\title{
ATIVIDADES DE FORMAÇÃO DO ENFERMEIRO NO ÂMBITO DA ATENÇÃ̂ BÁSICA À SAÚDE*
}

\author{
Viviane Caroline Medeiros ${ }^{1}$, Aida Maris Peres ${ }^{2}$
}

\footnotetext{
${ }^{1}$ Mestranda do Programa de Pós-Graduação em Enfermagem da Universidade Federal do Paraná (UFPR). Bolsista CAPES. Paraná, Brasil. E-mail: vivianecaroline@uol.com.br

${ }^{2}$ Doutora em Enfermagem. Professora do Programa de Pós-Graduação de Enfermagem da UFPR. Paraná, Brasil. E-mail: amaris@ufpr.br
}

RESUMO: Este estudo teve como objetivo caracterizar oportunidades vivenciadas e atividades realizadas durante as aulas práticas e estágios na atenção básica durante a formação do enfermeiro. A pesquisa do tipo exploratória não probabilística teve coleta de dados no período de fevereiro a março de 2009, por aplicação de questionário semiestruturado a um grupo amostral de 231 acadêmicos matriculados no último ano do curso de graduação em enfermagem de seis Instituições de Ensino Superior de Curitiba-PR. Foram identificadas: realização de diversas atividades relacionadas às técnicas básicas de enfermagem, poucas oportunidades de atividades gerenciais, e que o enfoque principal das Instituições de Ensino Superior na atenção básica está voltado às atividades educativas. Os resultados sustentam discussões para a avaliação curricular e mudanças no ensino de enfermagem. Recomenda-se que as escolas propiciem a atuação dos acadêmicos no contexto multiprofissional e valorizem oportunidades de aprendizagem relacionadas ao trabalho do enfermeiro nas atividades de atenção básica.

DESCRITORES: Enfermagem. Atenção primária à saúde. Educação em enfermagem.

\section{ACTIVITIES IN NURSING EDUCATION IN THE PRIMARY HEALTH CARE ENVIRONMENT}

\begin{abstract}
The objective of this study was to characterize the opportunities and activities experienced during the practical classes and internship concerning primary care in formal Nursing Education. The exploratory data was researched between February and March of 2009, with the application of semi-structured questionnaire in a sample of 231 nursing students enrolled in the final two semesters at six undergraduate institutions in Curitiba, Brazil. We conclude that the development of several activities related to basic nursing techniques, limited managerial, and the primary focus of Institutions of Higher Education in Nursing on primary care is directed to educational activities. The results sustain discussion to assess curricular changes on nursing education in Brazil. We recommend that undergraduate institutions promote academic performance in a multi-professional context and value learning opportunities related to nursing work and primary care activities.
\end{abstract}

DESCRIPTORS: Nursing. Primary health care. Education, Nursing.

\section{ACTIVIDADES PARA LA FORMACIÓN DEL ENFERMERO EN LA ATENCIÓN PRIMARIA DE SALUD}

RESUMEN: El estudio tuvo como objetivo caracterizar las actividades de aprendizaje realizadas en las prácticas de la atención primaria de salud durante el pregrado de enfermería. La investigación es de carácter exploratorio, no probabilística. La recolección de los datos se hizo de febrero a marzo de 2009, mediante un cuestionario semi-estructurado aplicado a una muestra de 231 estudiantes matriculados en el último año de la carrera de enfermería, de seis instituciones de enseñanza superior de la ciudad de Curitiba, Brasil. Se identificó lo siguiente: la realización de distintas actividades relacionadas con las técnicas básicas de enfermería, pocas actividades de gestión, y el foco principal de las instituciones de enseñanza superior en la atención primaria de salud centrado en las actividades educativas. Los resultados apoyan argumentos para la evaluación y los cambios curriculares en la enseñanza de la enfermería. Es recomendable que las escuelas propicien experiencias en el contexto multiprofesional y valoren oportunidades de aprendizaje relacionadas al trabajo del enfermero en atención primaria.

DESCRIPTORES: Enfermería. Atención primaria de salud. Educación en enfermería.

* $1^{\circ}$ lugar Prêmio Ingrid Elsen.

Texto Contexto Enferm, Florianópolis, 2011; 20 (Esp): 27-35. 


\section{INTRODUÇÃO}

O Sistema Único de Saúde (SUS) é responsável pela assistência à saúde de cerca de 140 milhões de brasileiros. ${ }^{1}$ Atua nos três níveis de atenção à saúde, sendo eles: atenção básica, média e alta complexidade. Na atenção básica destacam-se as ações de promoção à saúde e prevenção de doenças, além de compor o primeiro nível de atendimento curativo. A média complexidade visa atender aos principais problemas de saúde e agravos da população, através de atendimentos com profissionais especializados, e a alta complexidade envolve alta tecnologia e alto custo.

Conforme o nível de atenção à saúde são organizados e otimizados o uso de recursos: básicos ou especializados, direcionados para a promoção, manutenção ou recuperação da saúde. A atenção básica tem sua relevância no SUS por ser a porta de entrada do usuário no sistema público de saúde e demanda ações de promoção, prevenção e cura de baixa complexidade com amplitude para resolver questões de saúde da maior parcela da população.

A atenção básica cumpre um papel estratégico na dinâmica de funcionamento do SUS porque estabelece relações contínuas com a população e por se desenvolver por meio de um trabalho em equipe que enfatiza práticas democráticas e participativas. ${ }^{1}$ Diante das diretrizes estabelecidas pelo SUS e da proposta de atenção básica, é necessário conhecer o papel do profissional enfermeiro e discutir a sua formação.

Neste contexto, as instituições de ensino têm buscado formas de abranger em seus currículos os conteúdos necessários para a formação dos profissionais que irão atuar no sistema de saúde, em condições para atuar e planejar suas ações e de suas equipes. Em geral, as propostas são fundamentadas nos princípios e diretrizes que regem a organização da saúde no Brasil.

A Enfermagem foi o primeiro segmento da saúde a avançar na discussão sobre a formação profissional para a atuação no SUS, sobretudo a partir dos encaminhamentos de propostas dos Seminários Nacionais sobre as Diretrizes Curriculares para o Ensino de Enfermagem (SENADEn). ${ }^{2}$ Como resultados, foram apontados caminhos para as mudanças na formação do enfermeiro voltada à atuação no sistema de saúde, priorizando as práticas de promoção e prevenção.

Parte-se do pressuposto que as oportunidades vivenciadas pelos concluintes dos cursos de graduação em Enfermagem de Curitiba, na aten- ção básica, se aproximam do preconizado pelas Diretrizes Curriculares Nacionais de Enfermagem (DCNs), ${ }^{3}$ no que se refere à formação para o mundo do trabalho no sistema de saúde brasileiro. No entanto, processos avaliativos de currículos pós-DCNs levam a repensar os paradigmas ideológicos que conduzem a formação, o enfermeiro que se pretende formar e seu compromisso ético, político e técnico diante da complexidade dos processos de trabalho em saúde e enfermagem. ${ }^{4}$

O foco deste estudo está nas possibilidades de aprendizagem na atenção básica durante a formação do enfermeiro. São consideradas as intersecções entre saúde e educação, com a adequação do ensino para a produção de conhecimentos e serviços com base nas necessidades sociais. ${ }^{5}$

Este estudo justifica-se pela importância do reconhecimento e caracterização das atividades proporcionadas para a formação do enfermeiro no contexto da atenção básica. A contribuição pretendida segue no sentido de sustentar discussões sobre acompanhamento e avaliação curricular que busquem relacionar a realidade da formação profissional do enfermeiro com as necessidades do sistema de saúde e as DCNs. Dessa forma, delineou-se a seguinte pergunta de pesquisa: quais as oportunidades de aprendizagem vivenciadas e as atividades realizadas na atenção básica pelos concluintes dos cursos de graduação em enfermagem de Curitiba-PR?

O enfermeiro é um profissional que assume compromisso com as políticas de saúde e com o planejamento das ações de promoção à saúde e prevenção de doenças. Assim, esta pesquisa tem como objetivo caracterizar as oportunidades de aprendizagem vivenciadas e as atividades realizadas durante as aulas práticas e estágios na atenção básica ao longo do curso de graduação em enfermagem.

\section{METODOLOGIA}

A abordagem escolhida para esta pesquisa foi quantitativa do tipo exploratória. Essa classificação foi realizada levando em conta o objetivo a ser alcançado. ${ }^{6}$

Como critério de inclusão de sujeitos, optou-se por acadêmicos matriculados nos dois últimos períodos do curso de graduação em enfermagem de Instituições de Ensino Superior (IESs) de Curitiba-PR. Sete IESs apresentavam acadêmicos nessas condições, porém uma delas optou por não participar do estudo. 
Assim, os locais da pesquisa foram seis IESs, que possuíam turmas concluintes do curso de graduação em Enfermagem no ano de 2009, e aceitaram participar do estudo. Dessas instituições, uma é pública e cinco são privadas, sendo três universidades e três faculdades. As universidades se diferenciam das faculdades pela obrigatoriedade legal de desenvolver também atividades de extensão e pesquisa. ${ }^{7}$ Quanto ao tempo de formação, cinco (83\%) instituições apresentam matrizes curriculares organizadas em oito semestres e uma (17\%) em 10 semestres.

Nos dias de aplicação do questionário estavam presentes e aceitaram participar da pesquisa 231 $(70,6 \%)$ acadêmicos matriculados no último ano do curso de graduação em enfermagem, caracterizando a seleção de sujeitos como técnica não probabilística. A população era de 327 estudantes, de acordo com os coordenadores das instituições estudadas. A amostra foi suficiente para responder a um poder estatístico de $80 \%$ e um nível de confiança de $95 \%$.

O questionário semiestruturado, com questões fechadas e abertas foi aplicado aos acadêmicos do último ano do curso de enfermagem das instituições. $\mathrm{O}$ instrumento abordou questões referentes às atividades realizadas durante a graduação na atenção básica, com enfoque principal nas práticas voltadas a esse nível de atenção. A coleta de dados foi realizada em horários pré-estabelecidos com os coordenadores das instituições no período de fevereiro e março de 2009.

Ressalta-se que a pesquisa atendeu os aspectos éticos contidos na Declaração de Helsinki e na Resolução 196/96 do Conselho Nacional de Saúde, incluindo o aceite em participar da pesquisa por meio de Termo de Consentimento Livre e Esclarecido (TCLE). O projeto foi aprovado pelo Comitê de Ética em Pesquisa do Setor de Ciências da Saúde da Universidade Federal do Paraná, sob os números do Registro CEP/SD: 653.190.08.12 e CAAE: 0075.0.091.000-08.

A tabulação dos dados coletados foi feita através do programa estatístico Epi Info versão 3.5.1, que proporciona a análise pela relação feita entre as questões de maior relevância do questionário, destacadas pelas autoras. Os resultados foram apresentados por estatística descritiva simples, disponibilizados pelo próprio programa a partir dessa escolha, após a alimentação e cruzamento dos dados.

Para a caracterização das oportunidades de aprendizagem foi realizada a descrição e posterior análise por meio de frequência simples das seguintes variáveis: gênero, faixa etária, IES, oportunidades extracurriculares, locais de aulas práticas e estágios, enfoque das aulas práticas e estágios, oportunidades de atuação em programas de saúde e atividades realizadas nas aulas práticas e estágios na atenção básica.

\section{RESULTADOS E DISCUSSÃO}

\section{Caracterização dos sujeitos}

Quanto ao gênero, 24 (10,4\%) sujeitos eram do sexo masculino e 207 (89,6\%) pertenciam ao sexo feminino. Assim, confirma-se o contexto histórico da Enfermagem marcado pelo predomínio da força de trabalho feminina em atividades que envolvem o cuidado no Brasil. Estes dados são corroborados por estudo que aponta a relação existente entre o fato de ser mulher e a opção pelos cursos de Enfermagem. ${ }^{8}$

Em relação à faixa etária, $125(54,1 \%)$ sujeitos estão entre 20 e 25 anos; 63 (27,3\%) de 26 a 30 anos; $33(14,3 \%)$ sujeitos de 31 a 40 anos; sete $(3,0 \%)$ entre 41 e 50 anos, e apenas um $(0,4 \%)$ acima de 50 anos. Dois sujeitos não responderam a essa questão.

Portanto, estes resultados indicam o predomínio de uma faixa etária jovem. Discute-se ${ }^{8}$ entre as vantagens da presença de acadêmicos mais jovens no curso de Enfermagem, a formação de enfermeiros com perspectivas mais precoces de oportunidades para o crescimento e progresso profissional, em contraposição à possível imaturidade para o enfrentamento dos desafios e compromissos da profissão.

Quanto às IESs, responderam ao questionário 43 acadêmicos da instituição $A, 71$ da instituição $B, 41$ da instituição $C, 10$ da instituição D, 38 da instituição E, e 28 da instituição F. Sobre os períodos do curso em que os sujeitos estão matriculados, 108 (46,8\%) acadêmicos estão no sétimo, $80(34,6 \%)$ no oitavo, $29(12,6 \%)$ no nono e $14(6,1 \%)$ no décimo período. Vale lembrar que todos os sujeitos são do último ano de graduação e que as diferenças ocorrem porque algumas instituições apresentam seu currículo composto por 8 semestres e outras por 10 semestres letivos.

\section{Oportunidades extracurriculares}

Na pesquisa, os sujeitos relatam que, além das oportunidades ofertadas na graduação, 166 sujeitos $(71,9 \%)$ participaram de atividades extracurriculares. As DCNs ${ }^{3}$ reforçam a necessidade dos discentes buscarem oportunidades de aprendizado extracurricular quando sugerem que as instituições 
de ensino incorporem as atividades complementares na carga horária de formação acadêmica em um percentual de $5 \%$ da carga horária total do curso.

É provável que grande parte dos acadêmicos dos últimos períodos do curso de enfermagem tenha buscado outras oportunidades de aprendizado além das atividades curriculares ofertadas na graduação para compor o percentual de atividades complementares exigidas pelos seus cursos. Isto mostra que as DCNs brasileiras apresentam uma estratégia adequada no sentido de uma formação mais completa, em convergência com o movimento desencadeado pelo Espaço Europeu de Educação Superior, ${ }^{9}$ que aponta diretrizes para uma formação clínica reflexiva a partir de diferentes possibilidades de aprendizagem.

A figura 1 mostra as atividades extracurriculares desenvolvidas pelos acadêmicos durante a graduação. Vale lembrar que algumas dessas oportunidades são procuradas pela necessidade do acadêmico aprimorar-se em técnicas e habilidades, que consideram ser lacunas de sua formação. A demonstração disso se dá pelo número de discentes que procuraram estágios remunerados e voluntários neste estudo.

Espera-se que grande parte dos discentes de universidades esteja inserida em projetos de pesquisa e extensão. Porém, foi observado que mesmo nessas instituições a importância dada a estes projetos não foi significativa, ficando, algumas vezes, em segundo lugar, após os estágios voluntários e remunerados. O acompanhamento pedagógico poderia ser realizado no sentido de orientar a distribuição de atividades extracurriculares relevantes à formação.

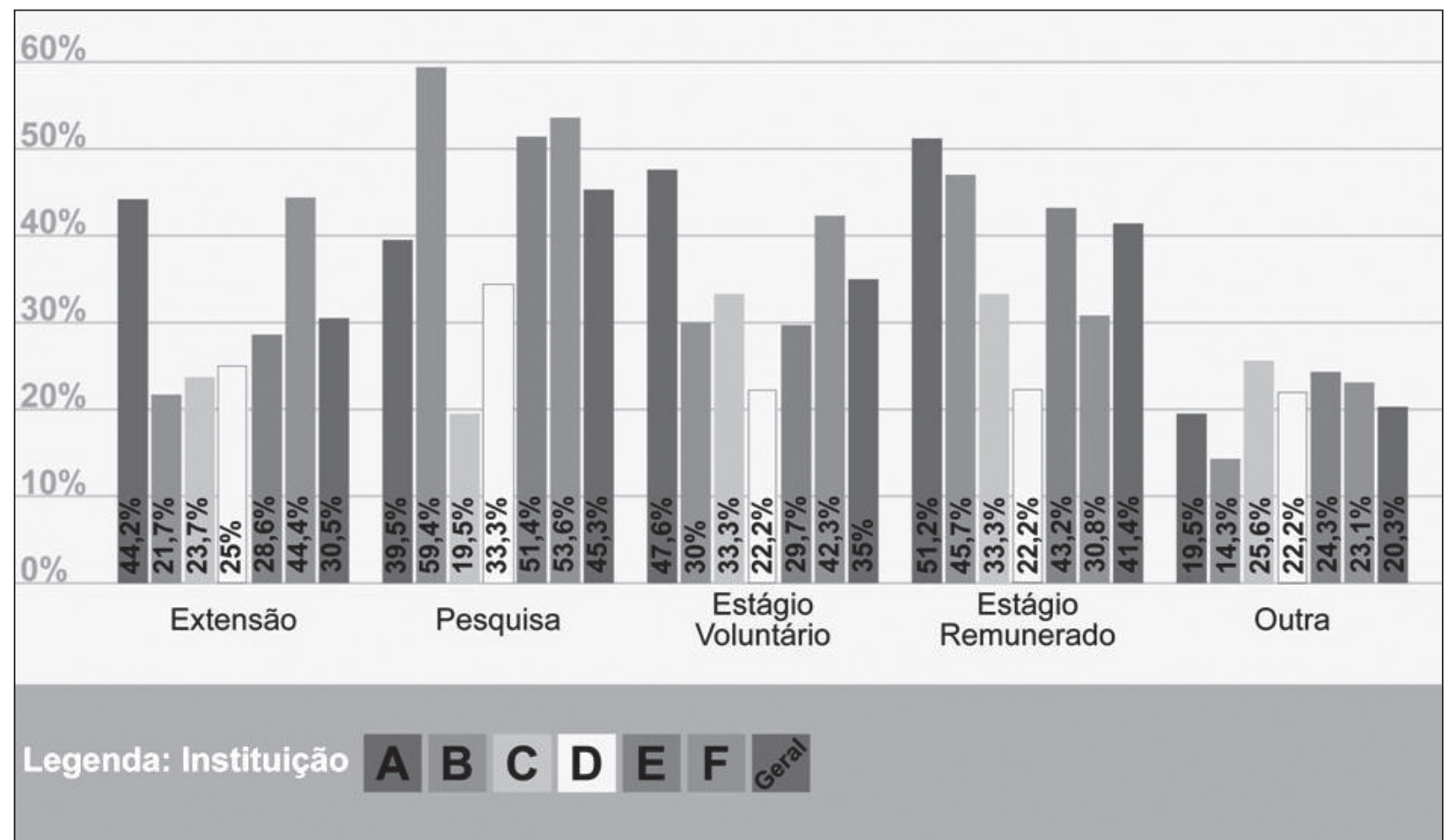

Figura 1 - Oportunidades extracurriculares vivenciadas por discentes de enfermagem de CuritibaPR, 2009

\section{Locais de aulas práticas e estágios}

O município de Curitiba, onde é realizada a maior parte dos estágios e aulas práticas das instituições pesquisadas na atenção básica, está dividido em nove Distritos Sanitários (DSs). São 51 Unidades Básicas de Saúde (UBSs) e 52 Unidades Saúde da Família (USFs). Além destas, o município conta ainda com nove Centros de Atenção
Psicossocial (CAPSs) e oito Centros Municipais de Urgências Médicas (CMUMs). ${ }^{10}$

Neste estudo, considera-se que as unidades de saúde de Curitiba que oferecem ações de atenção básica são as Unidades Básicas de Saúde e as Unidades Saúde da Família por desenvolverem ações programadas no nível primário de atenção à saúde. 
Tabela 1 - Locais de realização de estágios e aulas práticas de enfermagem em Curitiba-PR, 2009

\begin{tabular}{|c|c|c|c|c|c|c|c|c|}
\hline \multirow[b]{2}{*}{ Instituição } & \multicolumn{2}{|c|}{ UBS } & \multicolumn{2}{|c|}{ USF } & \multicolumn{2}{|c|}{ Distrito sanitário } & \multicolumn{2}{|c|}{ Outro } \\
\hline & $\mathbf{n}$ & $\%$ & $\mathbf{n}$ & $\%$ & $n$ & $\%$ & $\mathbf{n}$ & $\%$ \\
\hline$A$ & 39 & $90,7 \%$ & 14 & $32,6 \%$ & 36 & $83,7 \%$ & 2 & $4,7 \%$ \\
\hline $\mathrm{B}$ & 70 & $98,6 \%$ & 63 & $93 \%$ & 21 & $29,6 \%$ & - & - \\
\hline $\mathrm{C}$ & 35 & $85,4 \%$ & 38 & $92,7 \%$ & 10 & $24,4 \%$ & - & - \\
\hline $\mathrm{D}$ & 9 & $100 \%$ & 3 & $33,3 \%$ & 2 & $22,2 \%$ & - & - \\
\hline $\mathrm{E}$ & 34 & $91,9 \%$ & 27 & $73 \%$ & 3 & $8,1 \%$ & 1 & $2,7 \%$ \\
\hline $\mathrm{F}$ & 27 & $96,4 \%$ & 12 & $42,9 \%$ & - & - & 1 & $3,6 \%$ \\
\hline Total & 214 & $93,4 \%$ & 157 & $69,9 \%$ & 72 & $31,4 \%$ & 4 & $1,7 \%$ \\
\hline
\end{tabular}

Ao relacionar as respostas dos sujeitos com o cenário do estudo descrito no parágrafo anterior, cogita-se que as aulas práticas e estágios na atenção básica estão distribuídos em unidades de saúde de diferentes características. As unidades de saúde que atuam com enfoque de Estratégia de Saúde da Família apresentam uma composição de equipes mais estruturadas quanti e qualitativamente, ${ }^{10}$ o que favorece o entendimento do discente sobre o sistema de saúde e o trabalho da equipe multidisciplinar.

Quanto à informação de que alguns acadêmicos não tiveram oportunidade de realizar atividades em alguns tipos de serviços na rede municipal de saúde, ressalta-se a possibilidade de que estes ainda as realizem durante o último ano, uma vez que os questionários foram aplicados no início do semestre, atingindo os discentes antes mesmo de cursarem algumas disciplinas, inclusive as relacionadas à organização e administração dos serviços de saúde, de acordo com cada currículo,

Durante a tabulação dos dados, foi identificado que alguns acadêmicos ainda têm dificuldade de entendimento sobre o conceito de atenção básica. Isso foi evidenciado devido às respostas no item "outros locais", quando surgiram clínicas particulares e hospitais.

\section{Enfoque das aulas práticas e estágios em atenção básica}

As aulas práticas em atenção básica podem apresentar diferentes enfoques, de acordo com a proposta da IES demonstrada na figura 2.

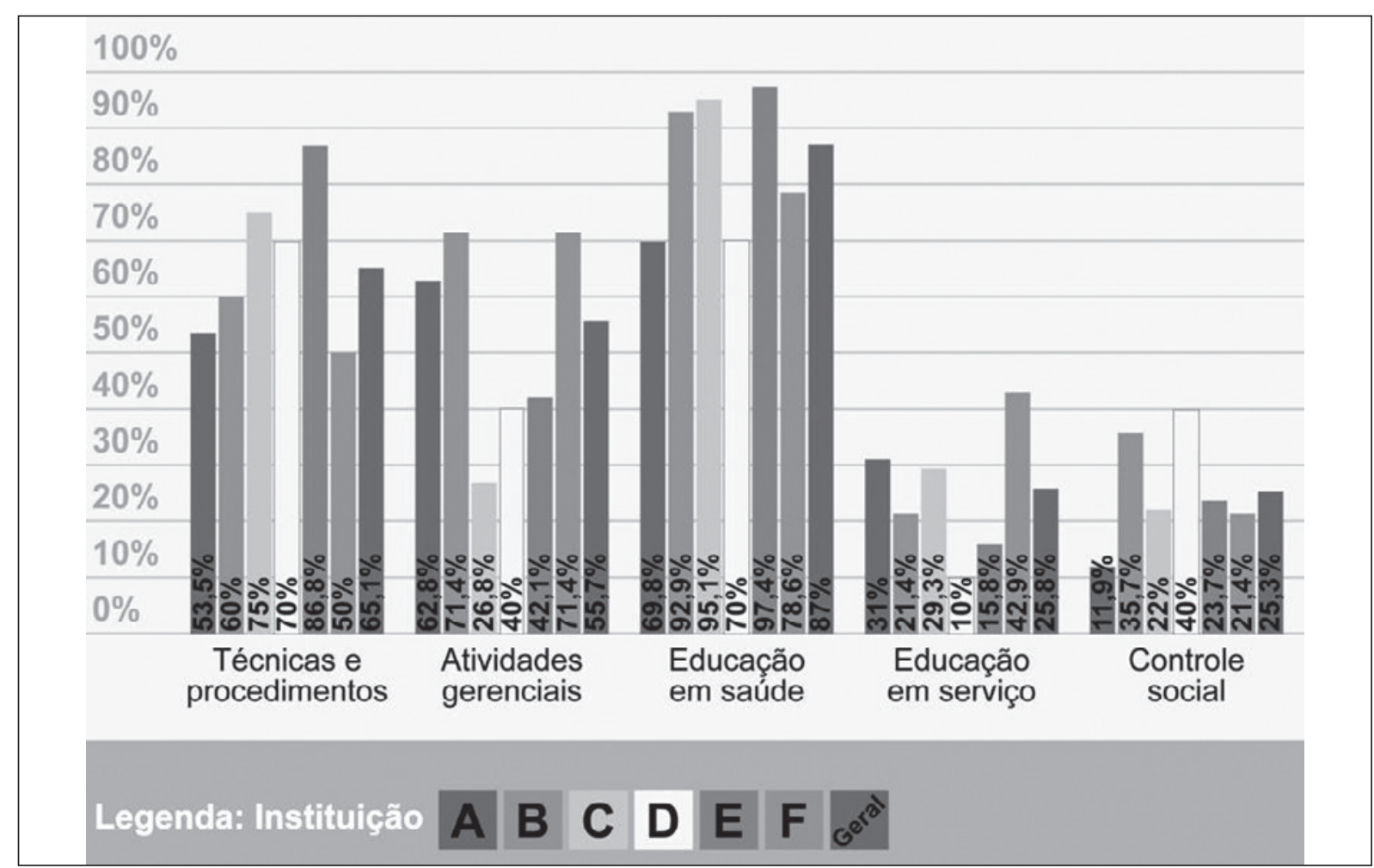

Figura 2 - Enfoque das aulas práticas e estágios em atenção básica dos cursos de enfermagem de Curitiba-PR, 2009 
Nota-se que o enfoque principal dado nas aulas práticas em atenção básica está relacionado às atividades educativas, com grande importância na educação em saúde. A educação em serviço tem pouco destaque nesse sentido, pois é visto que os acadêmicos trabalham mais com atividades de educação em saúde, por meio de reuniões, palestras e orientações aos usuários.

No estudo, as atividades gerenciais são evidenciadas no enfoque das aulas práticas e estágios, mas ainda ficam atrás das práticas de técnicas como vacinação, coleta de material para exames, consultas, entre outros. Entretanto, mostram-se irregulares enquanto oportunidades de aprendizagem propiciadas na formação pelas diferentes instituições.

Algumas suposições para que isto tenha acontecido sugerem que o ensino da gerência seja dissociado das outras disciplinas, pois em algumas instituições, esse tema é vivenciado apenas no último ano do curso. Ou ainda, que o ensino das disciplinas de administração aconteça prioritariamente com enfoque hospitalar. Estudo anterior aponta a importância da inserção do acadêmico de enfermagem nas equipes das unidades onde atuam, além de vivenciar atividades gerenciais em sua formação, como futuro coordenador da equipe de enfermagem. ${ }^{2}$

As atividades de controle social, cuja expectativa é que tenham grande relevância na formação, mostraram-se com pouca ênfase, com apenas 25,3\% dos acadêmicos identificando este enfoque nas aulas práticas. Como uma das diretrizes do Sistema Único de Saúde descrita na Lei 8.142,11 o controle social determina as ações de saúde já desde o nível local. A composição dos conselhos de saúde é formada por usuários (50\%), profissionais (25\%) e gestores (25\%), logo, esta é uma oportunidade para a formação do enfermeiro na dimensão política.

\section{Oportunidades de atuação em programas de saúde}

Esse item questionava sobre os principais programas de atenção básica que os acadêmicos tiveram oportunidade de conhecer durante as aulas práticas e estágios.

Tabela 2 - Programas de saúde vivenciados por acadêmicos de enfermagem de Curitiba-PR, 2009

\begin{tabular}{|c|c|c|c|c|c|c|c|c|c|c|}
\hline \multirow[t]{2}{*}{ Instituição } & \multicolumn{2}{|c|}{ Saúde mental } & \multicolumn{2}{|c|}{ Saúde da mulher } & \multicolumn{2}{|c|}{ Saúde da criança } & \multicolumn{2}{|c|}{$\begin{array}{c}\text { Hipertensão e } \\
\text { Diabetes }\end{array}$} & \multicolumn{2}{|c|}{ Outros } \\
\hline & $\mathbf{n}$ & $\%$ & $\mathbf{n}$ & $\%$ & $\mathbf{n}$ & $\%$ & $\mathbf{n}$ & $\%$ & $\mathbf{n}$ & $\%$ \\
\hline A & 34 & $79,1 \%$ & 42 & $97,7 \%$ & 40 & $93 \%$ & 27 & $62,8 \%$ & 8 & $18 \%$ \\
\hline $\mathrm{B}$ & 25 & $35,7 \%$ & 70 & $100 \%$ & 65 & $92,9 \%$ & 67 & $95,7 \%$ & 1 & $8,6 \%$ \\
\hline C & 41 & $100 \%$ & 41 & $100 \%$ & 39 & $95,1 \%$ & 36 & $87,8 \%$ & 13 & $31,7 \%$ \\
\hline $\mathrm{D}$ & 5 & $50 \%$ & 10 & $100 \%$ & 6 & $60 \%$ & 8 & $80 \%$ & 3 & $30 \%$ \\
\hline$E$ & 33 & $86,8 \%$ & 37 & $97,4 \%$ & 35 & $92,1 \%$ & 38 & $100 \%$ & 13 & $34,2 \%$ \\
\hline $\mathrm{F}$ & 24 & $85,7 \%$ & 27 & $96,4 \%$ & 26 & $92,9 \%$ & 25 & $89,3 \%$ & 9 & $32,1 \%$ \\
\hline Total & 162 & $70,4 \%$ & 227 & $98,7 \%$ & 211 & $91,7 \%$ & 201 & $87,4 \%$ & 47 & $22,6 \%$ \\
\hline
\end{tabular}

Considerando as práticas de atenção básica nas unidades de saúde (UBS ou USF), os acadêmicos tiveram a oportunidade de participar do acompanhamento de diversos programas nos quais a US está inserida. Na tabela 2 os programas são descritos de acordo com a porcentagem de discentes de cada instituição que teve oportunidade de presenciar os atendimentos de cada um deles.

O Programa de Saúde da Mulher apresenta maior participação dos acadêmicos nas aulas práticas e estágios, correspondendo a 98,7\% dos discentes. Isso pode ter acontecido em decorrência do acompanhamento pré-natal, além das consultas seguidas de coletas de exames preventivos para câncer de colo uterino.
Sobre o Programa de Saúde Mental, vale destacar que é possível que, apesar de estar descrito no enunciado da questão, os acadêmicos tenham confundido as práticas de atenção básica com as hospitalares. No cenário desta pesquisa é conhecido que algumas instituições trabalham a saúde mental curricular apenas no ambiente hospitalar.

As principais atividades relacionadas ao Programa Saúde da Criança são referentes à puericultura e vacinação. Nesses atendimentos, os discentes têm oportunidade de acompanhar, durante $o$ atendimento e a consulta de enfermagem, o crescimento e desenvolvimento da criança, a avaliação nutricional e outros sinais e sintomas relatados pelos pais ou responsáveis. 
O Programa de Hipertensão e Diabetes é o que apresenta maior demanda das unidades de saúde, constituindo-se no principal atendimento à saúde do adulto. Isso ocorre devido à grande prevalência desses agravos na população. Estudos demonstram essa prevalência em torno de 22,3\% a $43,9 \%$ na população adulta. ${ }^{12}$

No item outros, foram citados pelos acadêmicos o Programa de Saúde do Adolescente e o Programa de Saúde do Idoso, previstos pelo
Ministério da Saúde. Entretanto no município do estudo estes programas não estão implantados em todas as unidades de saúde.

\section{Atividades realizadas nas aulas práticas e estágios na atenção básica}

As atividades assistenciais e gerenciais realizadas nas aulas práticas e estágios na atenção básica são descritas na tabela 3.

Tabela 3 - Atividades realizadas nas aulas práticas e estágios na atenção básica em Curitiba-PR, 2009

\begin{tabular}{|c|c|c|c|c|c|c|c|c|}
\hline \multirow[t]{2}{*}{ Atividade } & \multicolumn{2}{|c|}{ Nenhuma } & \multicolumn{2}{|c|}{1 a 4 vezes } & \multicolumn{2}{|c|}{5 vezes ou mais } & \multicolumn{2}{|c|}{ Sem resposta } \\
\hline & $\mathrm{n}$ & $\%$ & $\mathbf{n}$ & $\%$ & $n$ & $\%$ & $\mathrm{n}$ & $\%$ \\
\hline Consulta de enfermagem & 23 & $9,9 \%$ & 108 & $46,7 \%$ & 87 & $37,6 \%$ & 13 & $5,6 \%$ \\
\hline Reunião de equipe & 65 & $28,1 \%$ & 108 & $46,7 \%$ & 42 & $18,1 \%$ & 16 & $6,9 \%$ \\
\hline Supervisão de equipe & 112 & $48,4 \%$ & 73 & $31,6 \%$ & 25 & $10,8 \%$ & 21 & $9,0 \%$ \\
\hline Relatórios & 40 & $17,3 \%$ & 75 & $32,4 \%$ & 101 & $43,7 \%$ & 15 & $6,4 \%$ \\
\hline Ações de territorialização & 18 & $7,7 \%$ & 139 & $60,1 \%$ & 55 & $23,8 \%$ & 19 & $8,2 \%$ \\
\hline Cuidado domiciliar & 20 & $8,6 \%$ & 110 & $47,6 \%$ & 85 & $36,7 \%$ & 16 & $6,9 \%$ \\
\hline Recepção Acolhimento & 12 & $5,1 \%$ & 112 & $48,4 \%$ & 93 & $40,2 \%$ & 33 & $14,2 \%$ \\
\hline Farmácia & 32 & $13,8 \%$ & 112 & $48,4 \%$ & 74 & $32,0 \%$ & 13 & $5,6 \%$ \\
\hline Vacinas & 13 & $5,6 \%$ & 88 & $38,0 \%$ & 118 & $51,0 \%$ & 12 & $5,1 \%$ \\
\hline Injeções & 8 & $3,4 \%$ & 63 & $27,2 \%$ & 149 & $64,5 \%$ & 11 & $4,7 \%$ \\
\hline Curativos & 14 & $6,0 \%$ & 80 & $34,6 \%$ & 126 & $54,5 \%$ & 11 & $4,7 \%$ \\
\hline Inalação & 25 & $10,8 \%$ & 93 & $40,2 \%$ & 102 & $44,1 \%$ & 11 & $4,7 \%$ \\
\hline Coleta de exames & 50 & $21,6 \%$ & 106 & $45,8 \%$ & 56 & $24,2 \%$ & 19 & $8,2 \%$ \\
\hline Coleta de preventivo & 23 & $9,9 \%$ & 144 & $62,3 \%$ & 51 & $22,0 \%$ & 13 & $5,6 \%$ \\
\hline Sondagens & 94 & $40,6 \%$ & 93 & $40,2 \%$ & 30 & $12,9 \%$ & 14 & $6,0 \%$ \\
\hline Retirada de pontos & 74 & $32,0 \%$ & 114 & $49,3 \%$ & 30 & $12,9 \%$ & 13 & $5,6 \%$ \\
\hline
\end{tabular}

Total: $\quad n=231$

Dentre as atividades gerenciais, destaca-se a elaboração de relatórios. Reuniões e supervisão de equipe são pouco realizadas pelos acadêmicos, o que representa um déficit na formação do profissional que será coordenador da equipe de enfermagem e precisará realizar essas tarefas cotidianamente em sua atuação. Apesar disso, é importante lembrar que parte dos sujeitos ainda pode desenvolver essas atividades ao longo desse último ano na graduação.

Sabe-se o que trabalho de enfermagem, subdivide-se em vários processos de trabalho como cuidar e assistir, administrar e gerenciar, pesquisar e ensinar. O cuidar e o gerenciar são os processos mais evidenciados no trabalho do enfermeiro. ${ }^{4}$

No tocante às atividades assistenciais, a tabela 3 mostra que são realizados diversos procedimentos nas aulas práticas e estágios na atenção básica. As coletas de preventivos estão associadas ao Programa Saúde da Mulher, e as demais cole- tas de exames são laboratoriais. Em 2007, foram realizados 121.307 exames preventivos de câncer de colo de útero e 2.147.247 exames laboratoriais no município de Curitiba. ${ }^{13}$

Vacinas e injeções são os procedimentos mais realizados pelos acadêmicos, o que denota valorização da aquisição de habilidade técnica. Espera-se que estas atividades sejam abordadas para além do enfoque técnico na formação e relacionadas à programação das ações de saúde, como por exemplo, com o Programa Nacional de Imunização.

Recepção e acolhimento também são realizados pela maior parte dos acadêmicos. Também é questionado se o acolhimento é realizado em conjunto a uma discussão com base nas políticas de humanização.

As ações de territorialização, base para a programação das atividades de saúde e para a compreensão do conceito de território, área e 
micro-área de risco, foram atividades que a maioria dos discentes teve a oportunidade de realizar. Dessa forma, é possível afirmar que os acadêmicos participam de todas as atividades rotineiras das unidades de saúde.

Uma informação preocupante é a de que, de acordo com a tabela 3, 9,9\% dos discentes relataram não ter realizado nenhuma consulta de enfermagem. De acordo com a Lei do Exercício Profissional do Enfermeiro, a consulta de enfermagem é uma competência privativa do enfermeiro. ${ }^{14}$ A realização desta pressupõe o domínio pelos enfermeiros das habilidades de comunicação, observação e de técnicas; suas metodologias próprias permitem que o enfermeiro tenha, de fato, uma atuação definida no serviço de saúde. ${ }^{14}$

Esse dado levanta o questionamento sobre os motivos para esses acadêmicos não terem realizado consultas de enfermagem. Uma justificativa pode ser a dificuldade de orientação individualizada nas aulas práticas para a realização de consulta. Outra razão pode ser o enfoque diferenciado da instituição formadora, que prioriza outras atividades durante suas aulas.

As lacunas na formação refletem diretamente na atuação do enfermeiro observada no município. De acordo com os relatórios, no ano de 2007 foram realizadas 379.180 consultas de enfermagem, número reduzido quando comparado às 3.287.305 consultas médicas. ${ }^{13}$

\section{CONSIDERAÇÕES FINAIS}

A caracterização das atividades realizadas pelos discentes de Enfermagem nas aulas práticas e estágios na atenção básica permitiu identificar oportunidades e lacunas na formação do enfermeiro. Os resultados são considerados relevantes para discussões acerca da avaliação curricular, considerando como foco da formação o sistema de saúde brasileiro e as necessidades de saúde da população.

O estudo aponta a tendência, vinda das próprias instituições de ensino e estimulada pelas recentes discussões acerca desse tema, que o processo de formação em saúde se aproxime cada vez mais do Sistema Único de Saúde. As aulas práticas e estágios têm sido desenvolvidos em locais com diversos tipos de atendimentos, o que demonstra que as oportunidades de aprendizagem são valorizadas pelas IESs em seus currículos. Resta saber se há articulação entre as disciplinas para que a diversidade das oportunidades de aprendizagem não aconteça de forma fragmentada.
Quanto às oportunidades de aprendizado extracurricular, compreendidas principalmente como pesquisa, extensão e estágios voluntários e remunerados, a maioria dos acadêmicos relataram que foi possível desenvolver alguma dessas atividades. Isso demonstra que, além do interesse em atuar em projetos de pesquisa e extensão universitária, os acadêmicos procuram outras formas de ampliar seu aprendizado. No entanto, fica evidente que buscam a aquisição de habilidades técnicas.

Recomenda-se que as escolas propiciem a atuação dos alunos no âmbito de ações do trabalho multiprofissional, mas mantenham oportunidades de aprendizagem nas ações exclusivas do enfermeiro como consulta de enfermagem, planejamento, coordenação e avaliação dos programas de saúde e da equipe de enfermagem.

\section{REFERÊNCIAS}

1. Coelho IB. Os impasses do SUS. Ciênc Saúde Colet. 2007 Mar-Abr; 12(1):307-17.

2. Peres AM, Ciampone MHT, Wolff LD. Competências gerenciais do enfermeiro nas perspectivas de um curso de graduação de enfermagem e do mercado de trabalho. Trab Educ Saúde. 2008 Nov-Fev; 5(3):453-72.

3. Ministério da Educação (BR), Conselho Nacional de Educação. Resolução $N^{0}$ 3, de 07 de novembro de 2001. Diretrizes curriculares nacionais do curso de graduação em Enfermagem. Diário Oficial da República Federativa do Brasil, 09 Nov 2001. Seção 1. p.37.

4. Meira MDD, Kurcgant P. O ensino de administração na graduação: percepção de enfermeiros egressos. Texto Contexto Enferm. 2009 Out-Dez; 18(4):670-9.

5. Weirich CF, Munari DB, Mishima SM, Bezerra ALQ. O trabalho gerencial do enfermeiro na rede básica de saúde. Texto Contexto Enferm. 2009 Abr-Jun; 18(2):249-57.

6. Gil AC. Como elaborar projetos de pesquisa. $4^{\mathrm{a}}$ ed. São Paulo (SP): Atlas, 2002.

7. Brasil. Decreto $N^{\circ} 5.773$, de 09 de maio de 2006. Dispõe sobre o exercício das funções de regulação, supervisão e avaliação de instituições de educação superior e cursos superiores de graduação e sequenciais no sistema federal de ensino.

8. Brito AMR, Brito MJM, Silva PAB. Perfil sociodemográfico de discentes de enfermagem de Instituições de Ensino Superior de Belo Horizonte. Esc Anna Nery Rev Enferm. 2009 Abr-Jun; 13(2):32833.

9. Sanjuan Quiles A, Martínez Riera JR. Nuevo enfoque en el proceso de enseñanza-aprendizaje para la interrelación de conocimientos y formación de clínica/comunitaria. Invest Educ Enferm. 2008 Set; 26(2supl):150-9. 
10. Curitiba. Secretaria Municipal de Saúde. Relatório anual de gestão [online]. Curitiba (PR): 2008. [acesso 2009 Jun 02]. Disponível em: http://www.curitiba.pr.gov.br/saude/sms/ RelatorioAnualdeGestaoSMSCuritiba2008.pdf

11. Ministério da Saúde (BR). Lei n. 8.142 de 28 de dezembro de 1990. Dispõe sobre a participação da comunidade na gestão do Sistema Único de Saúde (SUS) e sobre as transferências intergovernamentais de recursos financeiros na área da saúde e dá outras providências. Diário Oficial da República Federativa do Brasil, 28 dez 1990.
12. Sociedade Brasileira de Cardiologia. V Diretrizes Brasileiras de Hipertensão Arterial. Arquivos Brasileiros de Cardiologia. 2007 Set; 89(3):24-79.

13. Curitiba. Secretaria Municipal de Saúde. Acompanhamento do Plano Operativo Anual 2007. Relatório comparativo anual 2006 e 2007 [online]. Curitiba (PR): 2007 [acesso 2009 Jun 02]. Disponível em: www.curitiba.pr.gov.br/saude/ sms/relat\%F3rio\%20anual\%202006_2007.pdf

14. Brasil. Lei No 7.498, de 25 de junho de 1986. Dispõe sobre a regulamentação do exercício da Enfermagem e dá outras providências. Diário Oficial da República Federativa do Brasil, 26 Jun 1986. Seção 1. 